\title{
Docteur, j'ai inhalé le capuchon de mon inhalateur !
}

\section{Doc, I inhaled the Cup of my Ventoline'Spray!}

\author{
J. Smadja $\cdot$ N. Baarir $\cdot$ P. Ray
}

Reçu le 21 septembre 2017; accepté le 28 novembre 2017

(C) SFMU et Lavoisier SAS 2017

Un patient de 26 ans est amené par les pompiers au SAU pour « probable inhalation du capuchon de son inhalateur de Salbutamol ». Le patient explique être asthmatique et prendre du Salbutamol régulièrement en cas de dyspnée. Une heure auparavant, alors qu'il avait pris une bouffée, il a ressenti une forte douleur dans la poitrine concomitante d'une quinte de toux. La douleur s'est ensuite estompée, mais persiste une gêne au niveau de l'hémithorax droit et une toux sèche douloureuse.

À l'arrivée, il est eupnéique, hémodynamiquement stable, sa $\mathrm{SpO}_{2}$ est de $98 \%$ en air ambiant. L'auscultation cardiopulmonaire est sans particularité. Devant la pauvreté de l'examen clinique, et après avoir objectivé cette toux sèche et douloureuse, une radiographie de thorax de face est réalisée (Fig. 1A). Elle retrouve un corps étranger ovalaire, radioopaque dans la bronche souche droite. Il ne s'agit manifestement pas du capuchon de son inhalateur.

Le patient est alors adressé en fibroscopie pour ablation de ce corps étranger, qui s'avérera être une pièce de 10 centimes (Fig. 1B). Le patient expliquera par la suite que son inhalateur se trouvait dans la poche de son pantalon (visiblement sans son capuchon), et qu'une pièce de monnaie s'est a priori logée dans la fente du spray. Après ablation du corps étranger intrabronchique, le patient est rentré au domicile avec pour recommandation de bien reboucher son inhalateur après usage.

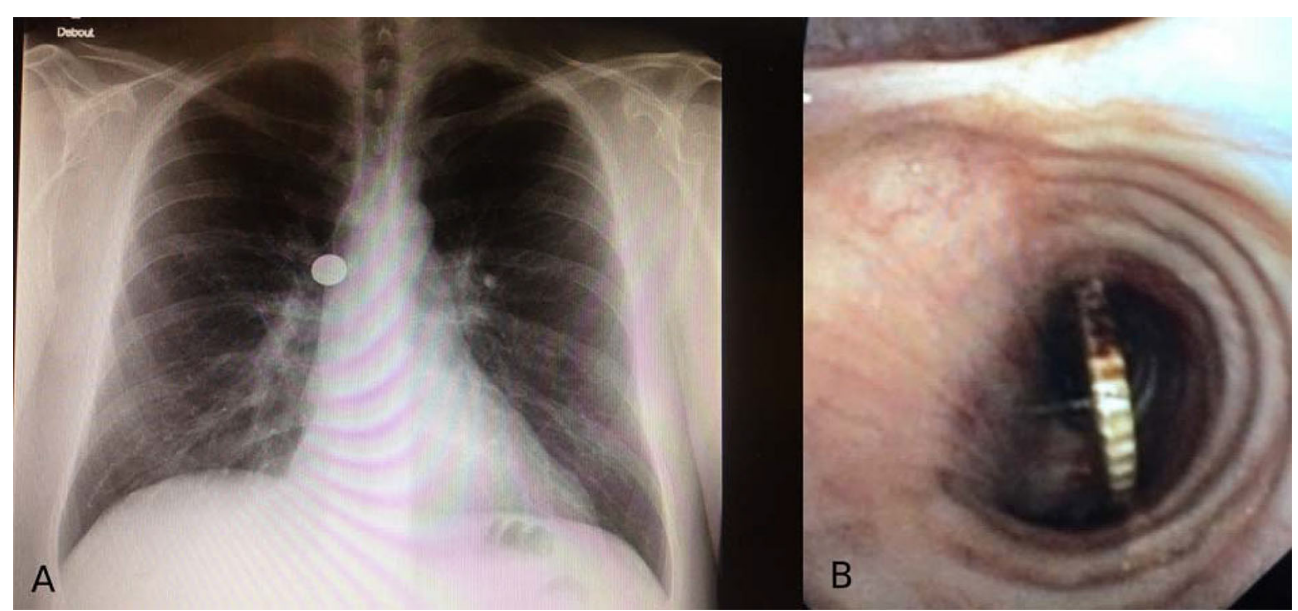

Fig. 1 A : opacité arrondie « métallique » dans la bronche souche droite ; B : mise en évidence d'une pièce de monnaie de 10 centimes en endoscopie bronchique

\footnotetext{
J. Smadja $\cdot$ N. Baarir · P. Ray $(\bowtie)$

Service accueil urgence, hôpital Tenon AP-HP, 55 rue Pelleport, 75020 Paris, France

e-mail : patrick.ray@tnn.aphp.fr

P. Ray

Sorbonne Universités UMPC université Paris 06, Paris, DHU

FAST (Fight Against STress)
} 